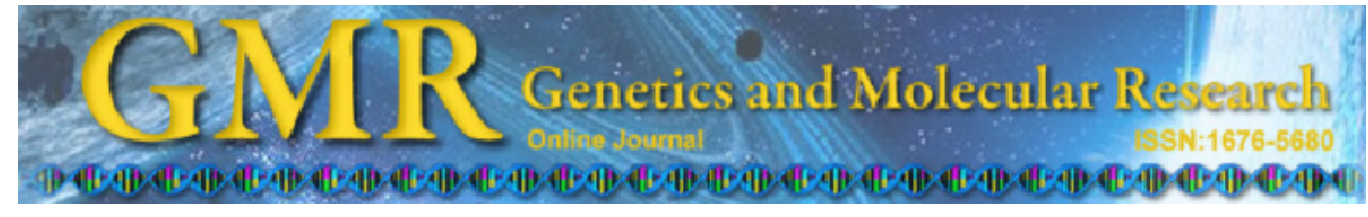

\title{
Heritabilities and genetic correlations for reproductive traits in an $F 2$ reciprocal cross chicken population
}

\author{
R.P. Savegnago ${ }^{1}$, M.E. Buzanskas ${ }^{1}$, B.N. Nunes ${ }^{1}$, S.B. Ramos ${ }^{1}$, \\ M.C. Ledur ${ }^{2}$, K. Nones ${ }^{3}$ and D.P. Munari ${ }^{1}$ \\ ${ }^{1}$ Departamento de Ciências Exatas, \\ Faculdade de Ciências Agrárias e Veterinárias, \\ Universidade Estadual Paulista "Júlio de Mesquita Filho", \\ Jaboticabal, SP, Brasil \\ ${ }^{2}$ Embrapa Suínos e Aves, Concórdia, SC, Brasil \\ ${ }^{3}$ Laboratório de Biotecnologia Animal, \\ Escola Superior de Agricultura "Luiz de Queiroz", \\ Universidade de São Paulo, Piracicaba, SP, Brasil \\ Corresponding author: D.P. Munari \\ E-mail: danisio@fcav.unesp.br
}

Genet. Mol. Res. 10 (3): 1337-1344 (2011)

Received September 8, 2010

Accepted February 16, 2011

Published July 5, 2011

DOI 10.4238/vol10-3gmr1053

ABSTRACT. Studies estimating genetic parameters for reproductive
traits in chickens can be useful for understanding and improvement
of their genetic architecture. A total of 1276 observations of fertility
(FERT), hatchability of fertile eggs (HFE) and hatchability of total eggs
(HTE) were used to estimate the genetic and phenotypic parameters of
467 females from an F2 population generated by reciprocal crossing
between a broiler line and a layer line, which were developed through
a poultry genetics breeding program, maintained by Embrapa Swine
and Poultry, Concordia, Santa Catarina, Brazil. Estimates of heritability
and genetic and phenotypic correlations were obtained using restricted
maximum likelihood calculations under the two-trait animal model,
including the fixed effect of group (hatching of birds from the same
genetic group) and the random additive genetic and residual effects.
The mean percentages for FERT, HFE and HTE were $87.91 \pm 19.77$,
$80.07 \pm 26.81$ and $70.67 \pm 28.55 \%$, respectively. The highest heritability
estimate $\left(\mathrm{h}^{2}\right)$ was $0.28 \pm 0.04$ for HTE. Genetic correlations for FERT 
with HFE $(0.43 \pm 0.17)$, HFE with HTE $(0.98 \pm 0.02)$ and FERT with HTE $(0.69 \pm 0.10)$ were positive and significant. Individuals with high breeding value for HTE would have high breeding values for HFE and FERT because of the high genetic association between them. These results suggest that HTE should be included as a selection criterion in genetic breeding programs to improve the reproductive performance of chickens, because HTE had the highest heritability estimate and high genetic correlation with FERT and HFE, and it is the easiest to measure.

Key words: Genetic association; Fertility; Hatchability; Heritability; White leghorn

\section{INTRODUCTION}

In chicken strains undergoing selection, both sires and dams contribute towards productive performance traits, but selection must be done with greater emphasis on dams if the objective of selection is to improve the performance of reproductive traits. Therefore, evaluating and understanding the genetic architecture of reproductive traits are of prime importance in providing females for mating and consequently improving these traits.

It has been reported that reproductive traits in chickens have low estimates of heritability and are strongly influenced by the environment and by nonadditive effects of genes. Selection for fertility and hatchability is based on a hypothesis of selection against segregation of deleterious recessive genes, probably at low frequencies, with a presumption that the heritabilities are low (Gowe et al., 1993). Thus, phenotypes are not good indicators of the best breeding values for reproductive traits; in this case, family selection is more recommended than individual selection (Rishell, 1997).

The relationships between productive traits (body weight, egg size and egg production) and reproductive traits (fertility and hatchability) are of interest because if the relationships are strong, they may affect progress of selection. Selection for productive traits could affect the performance of reproductive traits and might directly affect commercial performance, as in the case of meat chickens, for which hatchability is one of the most important attributes of parent stock performance (Hunton, 1971).

Fertility is defined as the interaction between maternal and paternal gametes to produce a viable zygote and can be expressed as the number of fertile eggs per chicken. Chicken selection for rapid growth can affect fertility by increasing the frequency of defective sperms and ova. Such defects are attributed to neuroendocrine imbalances, disruption of gametogenesis synchrony, dysfunctions of ovulation-oviposition patterns, and reduced libido (Barbato et al., 1984).

The hatchability of fertile eggs is the ratio between fertile eggs that produce a viable chicken and all fertilized eggs. Hatchability is the composite of the embryos' ability to survive and the maternal contribution towards embryo survival (Custódio, 1997). The hatchability of eggs that are laid is another important commercial reproductive trait; it is defined as the ratio between the total number of chickens born and the total number of eggs incubated.

We estimated genetic and phenotypic parameters of reproductive traits in an F2 chicken population arising from crossing a broiler line and a layer line, in order to better understand the genetic architecture of these traits. 


\section{MATERIAL AND METHODS}

\section{Description of the study population}

An F2 chicken population (TCTC and CTCT) was developed through reciprocal crossing of a broiler line (TT) with a layer line (CC). This was developed at the Brazilian National Research Center for Swine and Poultry (Embrapa Suínos e Aves, Brazil). The aim of this reciprocal crossing was to produce populations with segregated quantitative trait loci (QTL) for performance and carcass traits. The TT male broiler line has a genetic background of Cornish, Hampshire and White Plymouth Rock breeds; it has been selected to improve body weight, feed conversion, retail cut yield, breast weight, viability, fertility and hatchability, and to reduce abdominal fat. The CC is a pure White Leghorn line, selected for egg production, egg weight, feed conversion, hatchability, sexual maturity, fertility, viability and egg quality, and for reduced body weight.

Before the reciprocal crosses between the two lines for development of the F1 population, the TT and CC lines were under multi-trait selection for six and eight generations, respectively. Seven TT males were mated with seven CC females to generate seven F1 TC families, totaling around 50 chickens, and seven CC males were mated with seven TT females to generate seven F1 CT families, also totaling around 50 chickens. A total of seven TC males and 21 TC females (one male and three females from each full-sib family) and seven CT males and 21 $\mathrm{CT}$ females were chosen at random to be parents of the F2 population. The F1 chickens chosen for generating the F2 population were reared as broiler breeders. Each F1 male was mated with three non-related F1 females by means of artificial insemination to avoid inbreeding. Each F1 female produced around 100 F2 offspring in 17 hatches over eight months in 1999 and 2000, thus resulting in around $4000 \mathrm{~F} 2$ chickens (with equal numbers of each sex and of each mating, i.e., CTCT and TCTC). Some of these chickens ( 80 males and 570 females, half TCTC and half CTCT) were reared as broiler breeders to evaluate reproductive traits, including egg production, fertility, hatchability of fertile eggs, hatchability of total eggs set, and semen traits (volume, motility, concentration, viability, and abnormal sperm).

The pedigree data included 13 sires and 85 dams of the F1 generation and 467 females of the F2 generation, resulting in 565 birds. Each of the 467 F2 females had three records on average, thus resulting in 1276 observations. F1 sires had an average of 36 female offspring, with a minimum of 21 and a maximum of 54. F1 females had an average of 14 female offspring.

\section{Traits studied}

Mating between F2 males and F2 females generated eggs that were incubated over eight different periods. Fertility, hatchability of fertile eggs and hatchability of total eggs were measured in these eggs. These parameters were expressed as percentages and defined as: a) Fertility (FERT): the ratio between the number of fertile eggs at 18 days of incubation and the total number of eggs incubated. Each egg was evaluated to determine the fertile status by candling at transfer of eggs from the layer to the hatchery; b) Hatchability of fertile eggs (HFE): the ratio between the numbers of hatched eggs and fertile eggs at 18 days of incubation; c) Hatchability of total eggs (HTE): the ratio between the number of hatched eggs and the total number of eggs incubated. This trait can be calculated as FERT multiplied by HFE. 


\section{Statistical analysis}

Chickens in the F2 population with zero fertility and those with no records for HFE and HTE were excluded from the data set. The least-squares method with the SAS GLM procedure (SAS 9.1, SAS Institute, Cary, NC, USA) was used to define the fixed effects to be included in the mixed model $(\mathrm{P}<0.01)$. The fixed effect from the group consisted of chickens from the same hatching (1 to 8$)$ and same genetic group (TCTC or CTCT). The eight incubations occurred between February and November 2001. Four of these incubations had records from TCTC and CTCT and the other four only had TCTC records, with a total of 12 groups categorized into fixed effects. The normality of the residuals was investigated for each trait, and records with residual standard deviations above +3.5 and below -3.5 were excluded.

The variance-covariance estimates and genetic parameters were obtained by means of the restricted maximum likelihood method, using the two-trait animal model and the MTDFREML software (Boldman et al., 1995). The fixed effect from the group and the random additive genetic and residual effects were included in the two-trait animal model, described as:

$$
y=X \beta+Z a+\varepsilon
$$

(Equation 1)

where $y$ is the vector of observations, $X$ is the incidence matrix of fixed effects, $\beta$ is the vector of the solutions of fixed effects, $Z$ is the incidence matrix of genetic effects, $a$ is the vector of the solutions of genetic effects, and $\varepsilon$ is the random residual vector. Equation 1 expressed in matrix notation gives Equation 2:

$$
\left[\begin{array}{l}
y_{1} \\
y_{2}
\end{array}\right]=\left[\begin{array}{cc}
X_{1} & 0 \\
0 & X_{2}
\end{array}\right]\left[\begin{array}{l}
b_{1} \\
b_{2}
\end{array}\right]+\left[\begin{array}{cc}
Z_{1} & 0 \\
0 & Z_{2}
\end{array}\right]\left[\begin{array}{l}
a_{1} \\
a_{2}
\end{array}\right]+\left[\begin{array}{l}
e_{1} \\
e_{2}
\end{array}\right]
$$

where $y_{1}$ and $y_{2}$ are the two traits used in the model and $b_{1}$ and $b_{2}$ are the solutions for fixed effects for traits 1 and 2 associated with the values of the incidence matrix $X$. The genetic random effects $a_{1}$ and $a_{2}$ are associated with the incidence matrix $Z$, and $e_{1}$ and $e_{2}$ are residual random effects for traits 1 and 2 . The variance-covariance matrix of random effects in a twotrait animal model is expressed as Equation 3:

$$
\operatorname{Var}\left[\begin{array}{c}
a_{1} \\
a_{2} \\
e_{1} \\
e_{2}
\end{array}\right]=\left[\begin{array}{cccc}
A \sigma_{a_{1}}^{2} & A \sigma_{a_{1} a_{2}} & 0 & 0 \\
A \sigma_{a_{2} a_{1}} & A \sigma_{a_{2}}^{2} & 0 & 0 \\
0 & 0 & I \sigma_{e_{1}}^{2} & I \sigma_{e_{1} e_{2}} \\
0 & 0 & I \sigma_{e_{1} e_{2}} & I \sigma_{e_{2}}^{2}
\end{array}\right]
$$

where $\sigma_{a_{1}}^{2}$ and $\sigma_{a_{2}}^{2}$ are random genetic additive variances for traits 1 and $2 ; \sigma_{e_{1}}^{2}$ and $\sigma_{e_{2}}^{2}$ are random residual variances, and $\sigma_{a_{1} a_{2}}$ and $\sigma_{e_{1} e_{2}}$ are genetic and residual covariances, respectively. 


\section{RESULTS}

The phenotypic means for FERT, HFE and HTE in each line (CTCT and TCTC) are shown in Table 1. This population had high fertility and hatchability, with a higher CV for HTE than the CVs for FERT and HFE for the two lines.

Table 1. Phenotypic mean standard deviation (SD) and coefficient of variation (CV) for fertility (FERT), hatchability of fertile eggs (HFE) and hatchability of total eggs (HTE) in TCTC and CTCT, F2 chicken lines developed through reciprocal crossing of a broiler line (TT) with a layer line (CC).

\begin{tabular}{llcccccc}
\hline Traits & \multicolumn{3}{c}{ CTCT $(\mathrm{N}=315)$} & & \multicolumn{3}{c}{ TCTC $(\mathrm{N}=961)$} \\
\cline { 2 - 4 } & Mean & SD & CV $(\%)$ & & Mean & SD & CV (\%) \\
\hline FERT (\%) & 87.85 & 20.34 & 23.15 & & 87.93 & 19.59 & 22.28 \\
HFE (\%) & 85.01 & 21.51 & 25.30 & & 78.46 & 28.16 & 35.89 \\
HTE (\%) & 74.72 & 25.86 & 34.61 & 69.34 & 29.27 & 42.21 \\
\hline
\end{tabular}

Based on the $t$-test, there was no significance difference between the FERT means of the two lines $(\mathrm{P}=0.9509)$, but for HFE and HTE there were significant differences between the means of the two lines $(\mathrm{P}<0.01)$. The fixed effect from the group (incubation and genetic group) was significant $(\mathrm{P}<0.05)$ for all traits. FERT had the lowest heritability estimate and the additive genetic variance estimates ranged from 0.01 to 0.06 for FERT, HFE and HTE (Table 2).

Table 2. Estimates of variance components for additive genetic $\left(\sigma_{a}^{2}\right)$, environmental $\left(\sigma_{e}^{2}\right)$ and phenotypic $\left(\sigma_{p}^{2}\right)$ effects and heritability $\left(\mathrm{h}^{2}\right)$, for fertility (FERT), hatchability of fertile eggs (HFE) and hatchability of total eggs (HTE).

\begin{tabular}{lcccc}
\hline & $\sigma_{a}^{2}$ & $\sigma_{e}^{2}$ & $\sigma_{p}^{2}$ & $\mathrm{~h}^{2}$ \\
Traits & & 0.09 & 0.10 & $0.12 \pm 0.04$ \\
FERT & 0.01 & 0.13 & 0.18 & $0.27 \pm 0.04$ \\
HFE & 0.05 & 0.15 & 0.21 & $0.28 \pm 0.04$ \\
HTE & 0.06 &
\end{tabular}

Estimates of additive genetic, phenotypic and environmental correlations for the traits are shown in Table 3. The genetic associations between all the traits were positive. The environmental and phenotypic correlations were also positive, except between FERT and HFE, for which the estimates were close to zero.

Table 3. Estimates of genetic $\left(r_{\mathrm{a}}\right)$, environmental $\left(\mathrm{r}_{\mathrm{e}}\right)$ and phenotypic correlations $\left(\mathrm{r}_{\mathrm{p}}\right)$ between fertility (FERT), hatchability of fertile eggs (HFE) and hatchability of total eggs (HTE).

\begin{tabular}{lccc}
\hline Traits & $\mathrm{r}_{\mathrm{a}}$ & $\mathrm{r}_{\mathrm{e}}$ & $\mathrm{r}_{\mathrm{p}}$ \\
\hline FERT and HFE & $0.43 \pm 0.17$ & $-0.06 \pm 0.03$ & $0.02 \pm 0.03$ \\
FERT and HTE & $0.69 \pm 0.10$ & $0.52 \pm 0.02$ & $0.54 \pm 0.02$ \\
HFE and HTE & $0.98 \pm 0.02$ & $0.74 \pm 0.01$ & $0.80 \pm 0.02$ \\
\hline
\end{tabular}

\section{DISCUSSION}

To compare our results with other studies, it has to be taken into account that this study 
population originated through reciprocal crossing of two distinct lines, which contributed towards increasing the genetic variability in this population. Data from reciprocal cross populations are usually used for studies on inheritance and possible genetic and phenotypic associations of traits, as in this study, and not to conduct selection in genetic breeding programs. Thus, it was expected that these results for estimates of genetic parameters would be somewhat different from those in other studies.

The means for all the traits were similar to those of other studies. Ledur et al. (1994) found that mean FERT ranged from 81.46 to $90.94 \%$ in two chicken strains. Schmidt and Figueiredo (2005) reported mean FERT over five generations of 90.25 and 89.79 in two strains selected for egg production. Bennewitz et al. (2007) reported a mean of $86 \%$ for the same trait. The mean HFE reported by Schmidt et al. (1998) ranged from 67.27 to $86.97 \%$ in four broiler strains. Petek et al. (2005) reported means ranging between 72.31 and $90.63 \%$ and Schmidt and Figueiredo (2005) found mean HFEs of 88.97 and 88.66 in two selected strains. Bennewitz et al. (2007) reported a lower mean HFE $(48 \%)$ than what we found in our study (80.07). The means found for HTE in previous studies were $72.31 \%$ (Petek and Dikmen, 2004), 90.63\% (Petek et al., 2005) and 42\% (Bennewitz et al., 2007).

The significant differences $(\mathrm{P}<0.01)$ between the means for HTE and HFE (Table 2 ) of the two lines were attributed to sex-linked effects. The best performance for these traits was from the progeny of CTCT sires and CTCT dams. No references to sex-linked effects were found in the literature for comparison with the HTE and HFE data obtained in our study. Peters et al. (2008) found significant differences between the means for FERT and HFE, by studying different crosses between seven male and six female broiler lines. They observed that the means for FERT and HTE were significantly different, depending on the broiler cross evaluated.

Beaumont et al. (1997) reported heritability estimates for FERT of 0.09 from the sire component and 0.31 from the dam component. Sapp et al. (2004) found estimates for this trait ranging from 0.055 to 0.074 and Bennewitz et al. (2007) reported 0.07. These estimates were close to what we found in our study (0.12), except for the dam component heritability reported by Beaumont et al. (1997).

The heritability estimate for HFE in our study was higher than those found in previous reports. Kinney (1969) reported an $\mathrm{h}^{2}$ of 0.14 for this trait and Beaumont et al. (1997) found $\mathrm{h}^{2}$ $=0.05$ and 0.15 for the sire and dam components, respectively. Sapp et al. (2004) found estimates of $\mathrm{h}^{2}$ for HFE ranging from 0.06 to 0.07 and Bennewitz et al. (2007) found 0.14 for the same trait. It was difficult to find heritability estimates for HTE. Bennewitz et al. (2007) used Bayesian methodology to estimate heritability for HTE and found 0.13 for this trait.

It should be kept in mind that some differences between the heritability estimates in our study and those from other studies are due to different trait definitions, differences in the populations used and differences in the methodologies used to estimate genetic parameters. Until the mid-1990s, the method most often used for estimating genetic parameters was intraclass correlation among sire half-sibs. It was expected that lower heritability estimates would be found for HFE and HTE because reproductive traits have a low heritability estimate. The genetic parameter estimates in our study may have been increased because of the use of divergent strains in the reciprocal crosses.

These estimates may help to elucidate the genetic architecture of reproductive traits 
in future QTL studies. Some QTL studies have been performed on egg production, primarily using layer or broiler x layer crosses (Tuiskula-Haavisto et al., 2002; Wardecka et al., 2002; Sasaki et al., 2004; Schreiweis et al., 2006) and later using a layer strain crossed with a zoo-derived strain of red junglefowl (Wright et al., 2006), but there is a lack of studies incorporating meat quality, egg and reproductive traits using QTL analysis.

Jangarelli and Euclydes (2008) observed that there was greater efficiency in using marker-assisted selection when the traits had low heritability estimates. Research on molecular markers could be carried out and recommended for traits that are hard to measure or can only be measured in one sex (Schuman, 1990).

The highest genetic correlation was between HFE and HTE (0.98). The genetic correlation estimates between FERT, HFE and HTE were positive and favorable, thus indicating that any of these traits can be improved genetically by selecting for one of them. Ledur et al. (1994) reported estimates of genetic correlation between FERT and HFE of 0.39 and 0.16 in two broiler strains. Beaumont et al. (1997) found genetic correlation estimates between FERT and HFE of 0.71 and 0.59 , obtained from sire and dam components, respectively, and Sapp et al. (2004) reported estimates ranging from 0.73 to 0.97 . These genetic correlation estimates were close to our findings in the present study.

The three traits studied are usually considered to be in broiler reproductive performance evaluations in hatcheries. However, HTE is the product of FERT multiplied by HFE and partial-whole genetic correlations between HTE and FERT and between HTE and HFE were observed (Table 3). According to Fairfull and Gowe (1990), this effect occurs when one trait is composed of another. Because this, HTE had a strongly positive correlation with HFE and a positive correlation with FERT.

The medium to high positive environmental correlations (except between FERT and HFE, which was close to zero) indicated that these traits could be changed in the same direction by environmental changes, but that these gains would be lost in the next generation, through new combinations of genes. Some of the remaining hybrid vigor in the F2 generation could tend to reduce the environmental variation expressed in these traits.

Based on these results, we suggest that HTE should be included as a selection criterion in breeding programs, in order to improve the reproductive performance of chickens, because HTE had the highest heritability estimate and a high genetic correlation with FERT and HFE. Moreover, HTE is an easily measurable trait. Further research is needed to correlate these reproductive traits (which had low heritability estimates) with molecular markers, in order to improve chicken selection, increase the performance of reproductive traits and determine how HTE could affect performance traits.

\section{ACKNOWLEDGMENTS}

Research supported by FAPESP (Fundação de Amparo à Pesquisa do Estado de São Paulo) and Embrapa (Empresa Brasileira de Pesquisa Agropecuária). R.P. Savegnago and B.N. Nunes were recipients of scholarships from CAPES (Coordenação de Aperfeiçoamento de Pessoal de Nível Superior/Programa de Pós-Graduação em Genética e Melhoramento Animal - FCAV/UNESP); S.B. Ramos and K. Nones received scholarships from FAPESP and M.E. Buzanskas received a scholarship from CNPq (Conselho Nacional de Desenvolvimento Científico e Tecnológico). 


\section{REFERENCES}

Barbato GF, Siegel PB, Cherry JA and Nir I (1984). Selection for body weight at eight weeks of age. 17. Overfeeding. Poult. Sci. 63: 11-18.

Beaumont C, Millet N, Le Bihan-Duval E, Kipi A, et al. (1997). Genetic parameters of survival to the different stages of embryonic death in laying hens. Poult. Sci. 76: 1193-1196.

Bennewitz J, Morgades O, Preisinger R, Thaller G, et al. (2007). Variance component and breeding value estimation for reproductive traits in laying hens using a Bayesian threshold model. Poult. Sci. 86: 823-828.

Boldman KG, Kriese LA, Van Vleck LD and Van Tassell CP (1995). A Manual for Use of MTDFREML. A Set of Programs to Obtain Estimates of Variance and (co)variance (DRAFT) Lincoln. U.S. Department of Agriculture, Agriculture Research Service, Clay Center, Nebraska.

Custódio RWS (1997). Obtenção de repetibilidades na produção de ovos e pintos de um dia em galinhas para corte. Pesq. Agr. Bras. 32: 765-771.

Fairfull RW and Gowe RS (1990). Genetics of Egg Production in Chickens. In: Poultry Breeding and Genetics (Crawford RD, ed.). Elsevier Science Publishers, Amsterdam, 705-759.

Gowe RS, Fairfull RW, McMillan I and Schmidt GS (1993). A strategy for maintaining high fertility and hatchability in a multiple-trait egg stock selection program. Poult. Sci. 72: 1433-1448.

Hunton P (1971). Genetics of hatchability and its components and some production traits of chickens. Br. Poult. Sci. 12: 213-223.

Jangarelli M and Euclydes RF (2008). Endogamia e limite de seleção em populações sob seleção assistida por marcadores moleculares. Arq. Bras. Med. Vet. Zootec. 60: 1493-1501.

Kinney TB (1969). A Summary of Reported Estimates of Heritabilities and of Genetic and Phenotypic Correlations for Traits of Chickens. Agriculture Handbook, Washington.

Ledur MC, Schmidt GS, Figueiredo EAP, Ávila VS, et al. (1994). Parâmetros genéticos e fenotípicos em linhagens de aves selecionadas para corte. Pesq. Agr. Bras. 29: 503-508.

Petek M and Dikmen S (2004). The effect of prestorage incubation of quail breeder eggs on hatchability and subsequent growth performance of progeny. Anim. Res. 53: 527-534.

Petek M, Baspinar H, Ogan M and Balci F (2005). Effects of egg weight and lenght of storage period on hatchability and subsequent laying performance of quail. Turk. J. Vet. Anim. Sci. 29: 537-542.

Peters SO, Llori BM, Ozoje MO, Ikeobi CON, et al. (2008). Gene segregation effects on fertility and hatchability of pure and crossbred chicken genotypes in the humid tropics. Int. J. Poult. Sci. 7: 954-958.

Rishell WA (1997). Symposium: genetic selection - strategies for the future breeding and genetics - historical perspective. Poult. Sci. 76: 1057-1061.

Sapp RL, Rekaya R, Misztal I and Wing T (2004). Male and female fertility and hatchability in chickens: a longitudinal mixed model approach. Poult. Sci. 83: 1253-1259.

Sasaki O, Odawara S, Takahashi H, Nirasawa K, et al. (2004). Genetic mapping of quantitative trait loci affecting body weight, egg character and egg production in F2 intercross chickens. Anim. Genet. 35: 188-194.

Schmidt GS and Figueiredo EAP (2005). Selection for reproductive traits in white egg stock using independent culling levels. Braz. J. Poult. Sci. 7: 231-235.

Schmidt GS, Figueiredo EAP, Ledur MC and Munari DP (1998). Efeito da seleção quanto ao peso corporal nas características reprodutivas em linhagens de frangos de corte. Pesq. Agr. Bras. 33: 1595-1599.

Schreiweis MA, Hester PY, Settar P and Moody DE (2006). Identification of quantitative trait loci associated with egg quality, egg production, and body weight in an F2 resource population of chickens. Anim. Genet. 37: 106-112.

Schuman RM (1990). Genetic Engineering. In: Poultry Breeding and Genetics (Crawford RD, ed.). Elsevier Science Publishers, Amsterdam, 585-598.

Tuiskula-Haavisto M, Honkatukia M, Vilkki J, de Koning DJ, et al. (2002). Mapping of quantitative trait loci affecting quality and production traits in egg layers. Poult. Sci. 81: 919-927.

Wardecka B, Olszewski R, Jaszczak K, Zieba G, et al. (2002). Relationship between microsatellite marker alleles on chromosomes 1-5 originating from the Rhode Island Red and Green-legged Partrigenous breeds and egg production and quality traits in F(2) mapping population. J. Appl. Genet. 43: 319-329.

Wright D, Kerje S, Lundstrom K, Babol J, et al. (2006). Quantitative trait loci analysis of egg and meat production traits in a red junglefowl x white leghorn cross. Anim. Genet. 37: 529-534. 